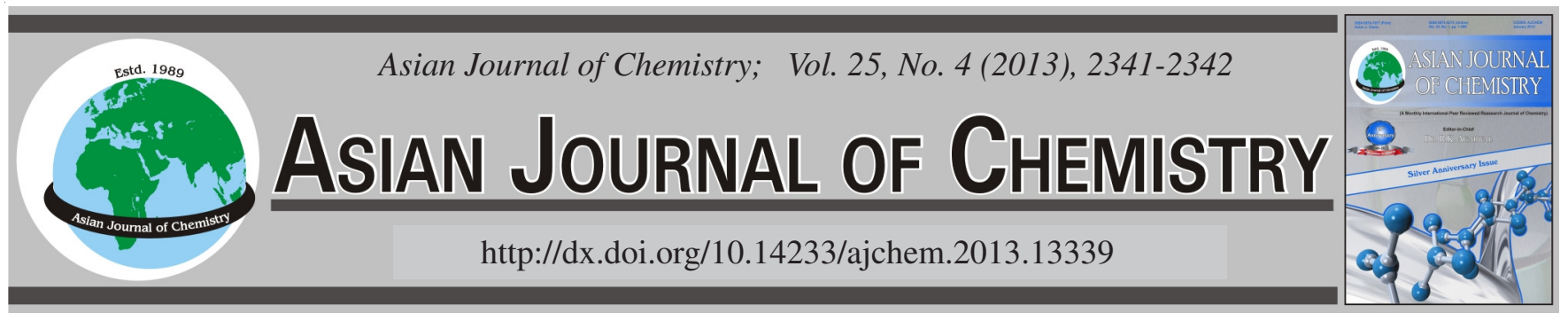

NOTE

\title{
Time-Resolved Transient Absorption Spectra of Gold Quantum Dot
}

\author{
LiNG XU ${ }^{1, *}$, YongAn SUN ${ }^{1}$ and WEN $\mathrm{LI}^{2}$
}

${ }^{1}$ College of Chemistry and Chemical Engineering, Inner Mongolia University for Nationalities, Tongliao 028043, P.R. China

${ }^{2}$ Technical Institute of Physics and Chemistry, The Chinese Academy of Sciences, Beijing 100080, P.R. China

*Corresponding author: E-mail: jdxuling1979@yahoo.com.cn; tlxuling1979@163.com

(Received: 27 December 2011;

Accepted: 29 October 2012)

AJC-12351

\begin{abstract}
Gold colloid was prepared by reducing aqueous solution of $\mathrm{HAuCl}_{4}$ in certain concentration with $\mathrm{NaBH}_{4}$ and gold particles with average size of $6 \mathrm{~nm}$ were used in this study. The time-resolved transient absorption spectra of the gold colloid were obtained by laser flash photolysis technique. The results showed that there was a broad and strong absorption band from 400 to $520 \mathrm{~nm}$ and a bleaching band in $520-850 \mathrm{~nm}$. At $472 \mathrm{~nm}$, the lifetime of gold colloid is about 38.6 microseconds ( $\mu \mathrm{s}$ ) according to the exponent function fitting on the decay curve. At the same time, we can learn from the transient absorption spectra that $\mu$ s-level gold quantum dot can be produced under $355 \mathrm{~nm}$ laser's excitation and it is basically attributed to the positive hole of gold quantum dot.
\end{abstract}

Key Words: Au colloid, Laser flash photolysis, Excited state lifetime.

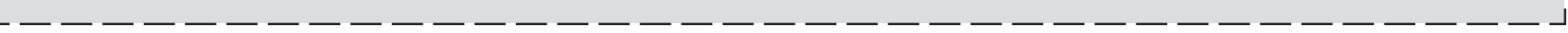

Recently, preparation and study of gold nano-particles has become a hot subject because of their chemical versatility, especially their application in catalysis and the diagnosis of some cancers ${ }^{1-4}$. Some proposed the mechanism that gold quantum dot with positive charge plays a key role in both homogeneous and heterogeneous catalysis ${ }^{2,4,5}$. We know that gold is a stable element in chemical reaction and it is difficult to get $\mathrm{Au}$ ion, let alone the active one in catalysis. In order to explore properties of Au ion, we set up two-step works i.e., (i) prepare Au quantum dot and (ii) then use laser flash photolysis to characterize Au quantum dot.

Firstly, Au colloids were prepared in our laboratory by the strong reducing method ${ }^{6,7}$. Both $\mathrm{HAuCl}_{4}$ solution with 4.8 $\times 10^{-4} \mathrm{M}$ and $4.8 \times 10^{-5} \mathrm{M}$ were prepared and then the strong reducing agent $\mathrm{NaBH}_{4}$ aqueous solution was dropped into $\mathrm{HAuCl}_{4}$ solution under stirring. Rapidly, Au quantum dot with different size and colour was formed respectively and all the Au quantum dot aqueous solutions were stable.

The TEM pictures in Fig. 1 show the average size of $\mathrm{Au}$ particles prepared by different concentrations of $\mathrm{HAuCl}_{4}$ was $12 \mathrm{~nm}$ and $6 \mathrm{~nm}$, respectively. Here we chose the light red Au colloid with average size $6 \mathrm{~nm}$ to carry out laser flash photolysis measurement. Apparently, we can not only produce Au quantum dot with unanimous nano-particle size, but also control the size of Au quantum dot by using different concentration of $\mathrm{HAuCl}_{4}$ solution in preparation.
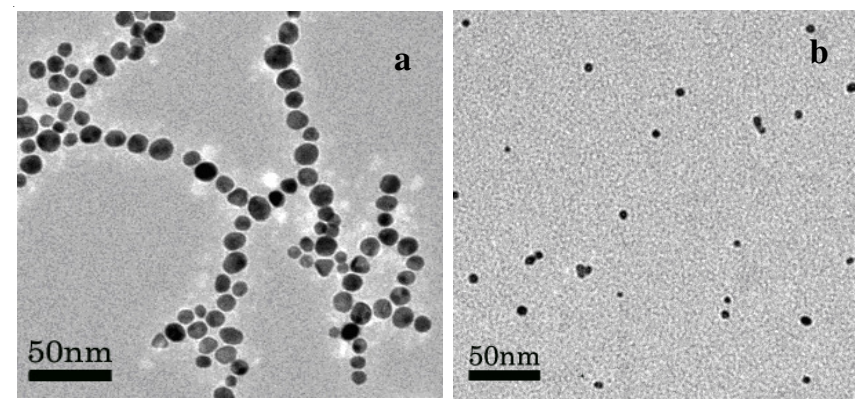

Fig. 1. TEM images of gold colloids (a) $\mathrm{CHAuCl}_{4}=4.8 \times 10^{-4} \mathrm{M}$ (b) $\mathrm{CHAuCl}_{4}=4.8 \times 10^{-5} \mathrm{M}$

Fig. 2 illustrates the time-resolved transient adsorption spectra. In this measurement, the $355 \mathrm{~nm}$ laser with $6 \mathrm{~ns}$ pulse width and $30 \mathrm{~mJ}$ energy/pulse was used as the excitation light and the probe light from Xe lamp was introduced into sample cell with right angle, all the signals were recorded with a Tektronix digital $100 \mathrm{MHz}$ TDS3012B oscilloscope ${ }^{8}$. From Fig. 2, it can be seen easily that there is a positive transient absorption band from 400 to $520 \mathrm{~nm}$ and a broad negative spectrum band (so-called bleaching spectrum band) with maximum peak of $650 \mathrm{~nm}$. As we expected, the obtained transient absorption spectra show that Au quantum dot really exists a kind of intermediate species within ms-level time domain, which naturally should be an active step and an active site during the reaction process. Here, the change of bleaching 
spectrum band intensity is opposite to that of the positive absorption band. In other words, the absorption spectrum intensity decreases while the bleaching spectrum intensity increases with time. To this case, the bleaching signal is a symbol of ground state electron which disappears step by step and the positive transient absorption signal is a symbol of electron excited state which forms from Au quantum dot. Of course, a difference between two spectrum bands can be found, i.e., the disappearing speed of the transient species is faster than the recovering speed of ground state. These results from the spectra provide important evidences to understanding the catalytic mechanism. In fact, the laser flash photolysis on $\mathrm{Au}$ quantum dot was carried out for the first time. Therefore, only the limited work could enable us to see its important significance and open up investigation field afterward. Compared with the narrow and weak absorption band with peak near $420 \mathrm{~nm}$ of Pt quantum dot, the absorption spectra of Au quantum dot are both strong and more red-shift.

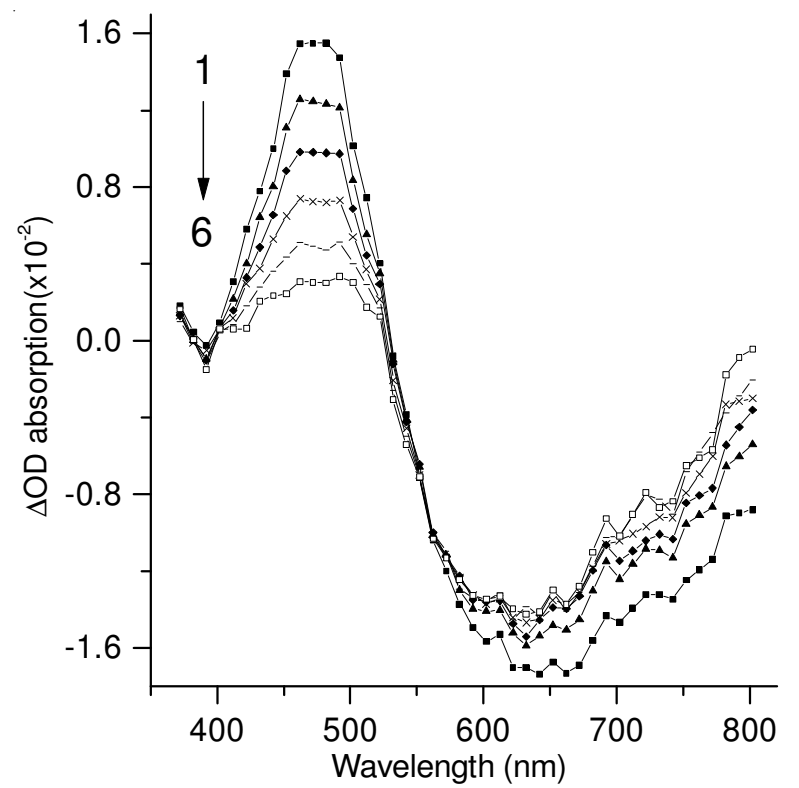

Fig. 2. Time-resolved transient adsorption spectra of Au quantum dot (with average size of $6 \mathrm{~nm}$ ) (1) 0.820 ; (2) 6.924 ; (3) 13.028 ; (4) 19.132; (5) 25.236 ; (6) $31.340 \mu \mathrm{s}$

According to these results, we consider possible mechanism as follows: Firstly, Au quantum dot is excited by a laser pulse and the exciton ( $\mathrm{e}^{-} \ldots \mathrm{h}^{+}$pair) is formed. Due to electronic movability, the excited electron migrates to the surface of $\mathrm{Au}$ quantum dot and then these electrons with negative charges may be neutralized by positive ions in solution. So, Au quantum dot becomes a quantum dot with positive charge $\left(\right.$ hole ${ }^{+}$). Usually, the hole in the quantum dot has longer lifetime. Hence, within microsecond range, we can observe its transient absorption spectra. In other words, transient absorption spectra of Au quantum dot show the existence of Au quantum dot with a hole ${ }^{+}$as transient species under laser excitation. The lifetime of this species can be obtained from exponent function fitting of the decay curve at certain wavelength within absorption range (Fig. 3). Here the fitting result is $38.6 \mathrm{~ms}$, the microsecond scale lifetime shows that an intermediate species, the excitation state of Au quantum dot, has a typical lifetime of photochemical intermediate species. In addition, one thing we must mention is that the transient spectra do not come from hydrated electron or related species because of the different spectrum pattern.

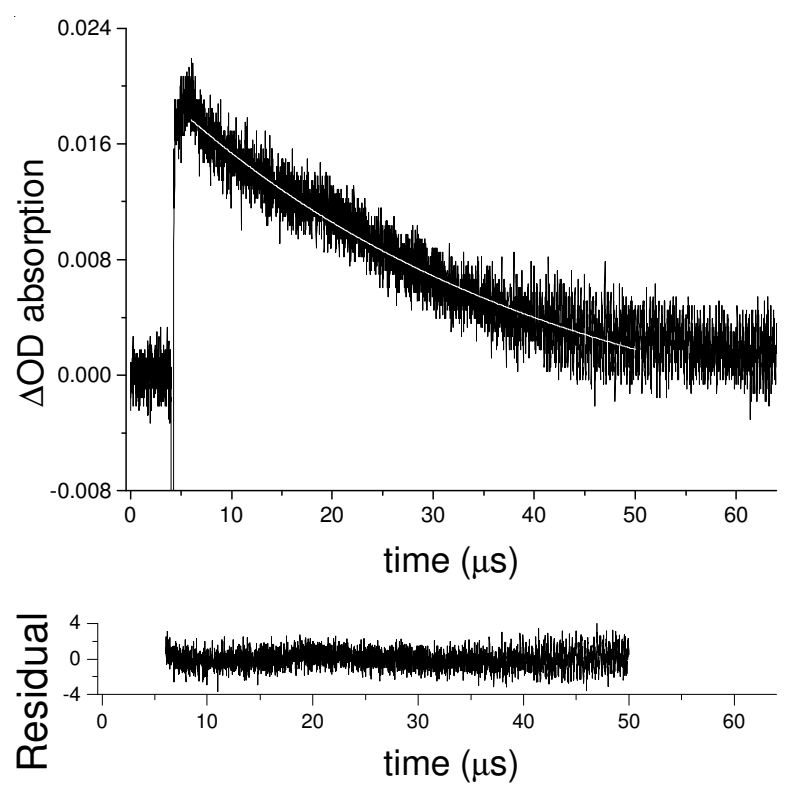

Fig. 3. Decay curve of Au quantum dot aqueous solution at $472 \mathrm{~nm}$ in laser flash photolysis

In summary, Au quantum dots with different size were prepared by $\mathrm{NaBH}_{4}$ reducing method. $6 \mathrm{~nm}$ Au quantum dot was chosen for laser flash photolysis and we get the Au quantum dot time-resolved transient absorption spectra for the first time. The result shows that there is an active species of Au quantum under $355 \mathrm{~nm}$ laser pulse and the lifetime of the active species is about $38 \mu$ s by exponent function fitting of decay curve at $472 \mathrm{~nm}$. Hence, this experimental evidence is really helpful to understanding the mechanism of Au quantum dot to be used as catalyst.

\section{ACKNOWLEDGEMENTS}

The authors thank the support of the Natural Science Foundation of Inner Mongolia (2011BS0203) and the Inner Mongolia Education Department (NJZY12121) and the Program for Young Talents of Science and Technology in Universities of Inner Mongolia Autonomous Region.

\section{REFERENCES}

1. J.D.S. Newman and G.J. Blanchard, Langmuir, 22, 5882 (2006).

2. A.M. Venezia, G. Pantaleo, A. Longo, G.D. Carlo, M.P. Casaletto, F.L. Liotta and G. Deganello, J. Phys. Chem. B, 109, 2821 (2005).

3. A. Lechtken, D. Schooss, J.R. Stairs, M.N. Blom, F. Furche, N. Morgner, O. Kostko, B.V. Issendorff and M.M. Kappes, Angew. Chem. Int. Ed., 46, 2944 (2007).

4. G. Lemière, V. Gandon, N. Agenet, J.P. Goddard, A. de Kozak, C. Aubert, L. Fensterbank and M. Malacria, Angew. Chem. Int. Ed., 45, 7596 (2006).

5. N. Marion and S.P. Nolan, Angew. Chem. Int. Ed., 46, 2750 (2007).

6. A.M. Gole, C. Sathivel and A. Lachke, J. Chromatogr. A, 848, 485 (1999).

7. R.G. DiScipio, Anal. Biochem., 236, 168 (1996).

8. J. Xue, W. Li, H. Yu, et al., Photograp. Sci. Phtochem., 24, 262 (2006). 